\title{
New developed formula to calculate the permittivity of ferrite-polymer composite
}

\begin{abstract}
A new developed formula named the Habashi-Zul formula, based on the Maxwell-Garnett (MG) formula was fabricated and used to calculate the complex permittivity of the Sm-YIG in PVDF composite samples in the frequency range of $10 \mathrm{MHz}$ to $1 \mathrm{GHz}$. The calculated permittivities results were compared with the measured values of each sample of Sm-YIG in PVDF composite samples and, with calculated results from various theoretical models including the MG, Looyenga, Bruggeman and, Sen Scala and Cohen. The Habashi-Zule formula presented higher accuracy as compared to other models.
\end{abstract}

Keyword: Ferrite-polymer composite; Maxwell Garnett; Permittivity; Sm-YIG-PVDF 